\title{
ARTICLE
}

\section{Geant4-based particle therapy simulation framework for verification of dose distributions in proton therapy facilities}

Takashi Akagi ${ }^{\mathrm{a}}$, Tsukasa Aso ${ }^{\mathrm{b} *}$, Go Iwai ${ }^{\mathrm{c}}$, Akinori Kimura ${ }^{\mathrm{d}}$, Satoru Kameoka ${ }^{\mathrm{e}}$, Se Beyong Lee ${ }^{\mathrm{f}}$, Yoshikazu Maeda ${ }^{\mathrm{g}}$, Naruhiro Matsufuji ${ }^{\mathrm{h}}$, Teiji Nishio ${ }^{\mathrm{e}}$, Chihiro Omachi ${ }^{\mathrm{i}}$, Seyjoon Park ${ }^{\mathrm{f}}$, Takashi Sasaki ${ }^{\mathrm{c}}$, Toshiyuki Toshito ${ }^{\mathrm{i}}$, Wataru Takase $^{\mathrm{e}}$, Tomohiro Yamashita ${ }^{\mathrm{e}}$ and Yoshiyuki Watase ${ }^{\mathrm{e}}$

${ }^{a}$ Hyogo Ion Beam Medical Center, 1-2-1, Kouto, Shinghu-cho, Ibo-gun, Hyogo 679-5165, Japan; ${ }^{b}$ Nagaoka University of Technology, 1603-1, Nagaoka, Niigata 940-2188, Japan; ${ }^{c}$ KEK, 1-1 Oho, Tsukuba, Ibaraki 305-0801, Japan; ${ }^{d}$ Ashikaga Institute of Technology, 268-1, Omae-cho, Ashikaga, Tochigi 326-8558, Japan; ${ }^{e}$ National Cancer Center Hospital East, 6-5-1 Kashiwanoha, Kashiwa, Chiba 277-8577, Japan; ${ }^{\dagger}$ National Cancer Center, 323 Ilsan-ro, Ilsandong-gu, Goyang-si, Gyeongii-do 411-769, Republic of Korea; ${ }^{g}$ Fukui Prefectural Hospital Proton Therapy Center, 2-8-1 Yotsuii, Fukui-shi, Fukui 910-8526, Japan; ${ }^{h}$ National Institute for Radiology Science, 4-9-1 Anagawa, Inage-ku, Chiba-shi, Chiba 263-8555, Japan; ${ }^{i}$ Nagoya Proton Therapy Center, 1-1-1 Hirate-cho, Kita-ku, Nagoya 462-8508, Japan

Geant4 is a software toolkit used to simulate the interaction of particles in matter. Monte Carlo simulation is useful in the design of particle treatment facilities and quality assurance of treatment plans. The particle therapy simulation framework (PTSIM) is a Geant4-based simulation framework for particle therapy validated using protons and carbon-ions. The PTSIM provides a common platform to model three Japanese proton and ion therapy facilities, as well as three facilities in other countries, allowing users who are not Geant4 experts to accurately and efficiently run Geant4 simulations for any of these pre-built configurations. While Geant 4 has been heavily and widely used in medical physics research, clinical applications remain limited as a result of problems involving of computational efficiency and usability by medical staff. The present paper describes the available functionality and the effort on further development of PTSIM in order to apply PTSIM in a straightforward manner to clinical applications. The details of these features of functionalities related to the digital imaging and communications in medicine (DICOM) interface in order to coordinate with hospital information systems and the parallelization interface for grid systems in order to achieve better turnaround time are reported. Keywords: Geant4; Monte Carlo; proton therapy; particle therapy; dose distribution; DICOM;
DICOM-RT; treatment plan; grid; PTSIM

\section{Introduction}

Geant4[1,2] is a software toolkit that is used to simulate the interaction of particles in matter and has been widely used in various fields from high-energy physics (HEP) to nuclear physics to space and medicine. Monte Carlo simulation using Geant4 takes into account the physics of particle interactions using theoretical models or experimental cross-section data. Therefore, Geant 4 is considered to be the most accurate method by which to calculate the dose in radiotherapy. Monte Carlo simulations can be helpful in the design of treatment facilities, improvement of treatment plans, and quality assurance of ongoing treatments. Verification of the validity of Geant4 for use in dose calculations for nozzle design and quality assurance has been reported previously [3-6].

*Corresponding author. Email: aso@nagaokaut.ac.jp
The particle therapy simulation framework (PTSIM) $[7,8]$ is a Geant4-based simulation framework for particle therapy validated using protons and carbon-ions. The PTSIM provides a common platform on which to model three Japanese proton and ion therapy facilities, as well as three facilities in other countries, allowing users who are not Geant4 experts to accurately and efficiently run Geant4 simulations for any of these pre-built configurations.

Although the PTSIM has been heavily used in medical physics research, clinical applications remain limited. In order to apply the PTSIM to clinical applications, the PTSIM should be coordinated with hospital information systems. Then, medical users can use treatment planning systems and alternative dose viewers for dose review by comparing dose distributions between the predications by the pencil beam algorithm [9] and Monte Carlo simulation.

In the present paper, we summarize the available 
functionality and current efforts to further develop the PTSIM in order to apply it to clinical applications in a straightforward manner. This includes a parallel execution environment using grid systems for compensating computational resources in the treatment facility. These required functionalities are related to the digital imaging and communications in medicine (DICOM) [10] interface and the Grid interface.

\section{Overview of the PTSIM}

The PTSIM was developed as a simulation framework for advanced radio therapy funded by the Japan Science and Technology Agency (JST) as part of a program of the Core Research for Evolutional Research and Technology (CREST), October 2003 to March 2010.

The PTSIM is a single software application for modeling a treatment port consisting of a beam delivery system and a treatment head with patient data obtained from CT images. The configuration of the treatment port is defined by a series of parameters. Online commands are provided for modifying such parameters. Therefore, users are not requested to develop program code.

\section{DICOM interface}

Hospital information systems typically manage all information in DICOM format. The PTSIM includes code for importing such information and automatically apply parameters to the configuration of the treatment port and the patient geometry with its registration.

\subsection{Patient geometry}

The patient scan information from the CT scanner is provided as CT images in DICOM format. This information represents the patient image as a contiguous set of voxels formed into a patient geometry. The scalar in each voxel is given in Hounsfield units (HUs), which correspond to the density information of the material. The PTSIM constructs a patient geometry by placing voxels filled with materials corresponding to HUs in CT images. Since the overall CT images form a box, the patient geometry often includes both the patient and some amount of air surrounding the patient. Optimization of the patient geometry based on CT data in Geant4 is discussed in [11].

The DICOM interface supports additional functions, such as complement lack slices, reformation of pixel spacing with user-defined arbitral size, extraction of actual patient geometry by replacing voxels of the support table with air, and trimming by removing a special zone from the patient geometry. The mass density of the material in each voxel is calculated according to the function in a lookup table. The step of density resolution can be degraded, which contributes to reducing the number of materials in the geometry in order to suppress memory consumption.

By default, the material in each voxel is assumed to be water, the mass densities of which vary according to the HU value. The PTSIM provides two options for material assignments for calculating dose-to-tissue.

The first option follows a conversion method proposed in [5,12-13], in which the HU space is divided into material groups. The elemental composition and weight are preserved within a group, whereas the mass density continuously increases as the HU increases. Since the HU value itself does not contain exact information about the material, this option is considered to be an approximate method representing the patient geometry for dose-to-tissue.

The second option uses segmentation data of organs to determine the material in the voxel according to a series of regions of interest (ROIs). The ROIs will be defined using automatic segmentation tools or medical physicists and will be recorded in the radio therapy extensions (RT) of the DICOM format (DICOM-RT). The dataset of the ROI in DICOM is referred to as the RT-Structure-Set. The PTSIM imports both CT images and ROI data in DICOM format and assigns a material to the voxel by referring to the HU value and the ROI identification number. Here, the matching information between the ROI identification number and the material name must be defined by the user. Figure 1 shows a schematic diagram of the patient geometry construction procedure using the second option.

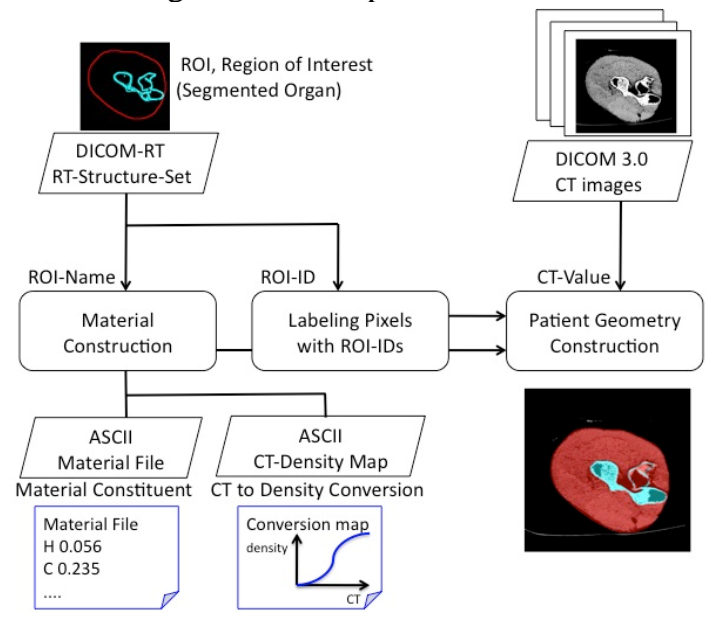

Figure 1. Schematic diagram of the patient geometry construction procedure using the second option.

\subsection{Beam delivery system configuration}

The treatment settings, such as the beam condition and the treatment head parameters, including patient registration, are determined in the treatment planning system. The dataset is referred to as RT-Plan or RT-IonPlan in DICOM format. In the PTSIM, the treatment settings should be imported in order to provide better usability and ensure the same condition for the treatment planning system and the proton treatment machine.

Although the statements of RT-Plan and RT-IonPlan are well established, the parameters might not have same meaning at different treatment facilities, because the 
components of the treatment ports are different. The current version of the PTSIM supports RT-Plan and RT-IonPlan at the Hyogo Ion Beam Medical Center (HIBMC) and the Fukui Prefectural Hospital Proton Therapy Center (FPHPTC), respectively.

\section{The PTSIM interface in the hospital information system}

We used Quality Assurance Control Interface (QACIF) software, which was developed as part of the Fukui Prefecture Advanced Research for Proton Therapy project at the FPHPTC. The QACIF is used to download a series of DICOM data, including RT-IonPlan and CT images, by specifying a patient identification number and a treatment identification number from the DICOM server, whereas the private information of the patient is made anonymous in order to ensure patient privacy.

After the PTSIM performs simulation for the patient data, the QACIF converts the final dose results and corresponding unit values in RT-Dose and RT-IonPlan formats and sent the results to the DICOM server. Figure 2 shows a schematic diagram of the hospital information system, which is relevant to the treatment plan and dose review at the FPHPTC. The upper and lower panels of the figure show the data flow for the clinical treatment plan and the data flow for the Monte Carlo-based treatment plan, respectively. $\mathrm{XiO}-\mathrm{N}$ is the software of the treatment planning system, which uses the K2Dose [14] algorithm for dose calculation. The final dose distributions are reviewed in $\mathrm{XiO}-\mathrm{N}$ and the other dose viewers in the hospital information system.

A similar interface program is available at the HIBMC, which has been developed at the HIBMC.

Treatment Planning System in Fukui Prefectural Hospital Proton Therapy Center

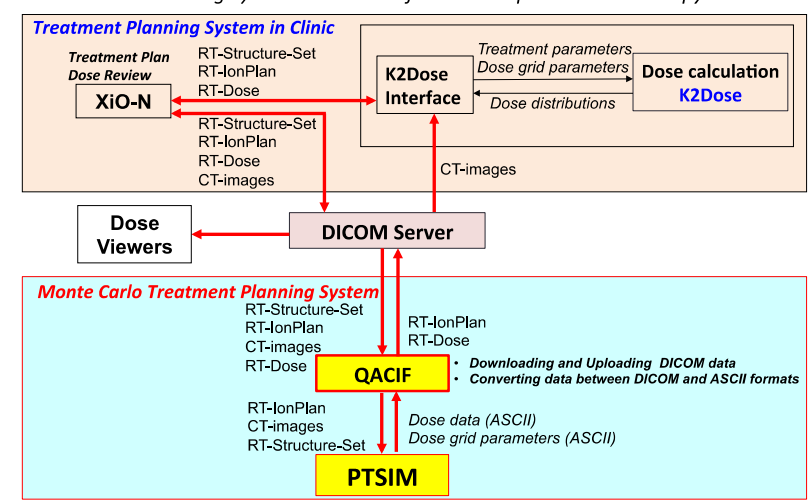

Figure 2. Treatment planning system at the FPHPTC

\section{Grid interface}

A treatment planning system calculates the dose based on the pencil beam algorithm and completes the dose estimation within five minutes. In contrast to the pencil beam algorithm, Monte Carlo simulation must simulate more than $9 \times 10^{8}$ primary protons in proton therapy simulation in order to achieve results with sufficient statistics. Therefore, the parallelization of simulation is essential for high-performance computing resources, such as computing clusters or grid-distributed computing environments. For the purpose of compensating the shortage of computing resources and reducing maintenance costs, we developed the universal grid interface (UGI) in order to perform simulations in multi-grid environments.

The UGI is a package that is included with Python and provides a command line interface for job submission, file manipulation, and monitoring in multi-grid middleware environments. The UGI is developed on top of the simple API of the grid application (SAGA)[15] framework, the resource namespace service (RNS)[16], and the XML-RPC[17]. The UGI supports a Web interface for job submissions, job monitoring, and simple dose visualization.

The performance of the PTSIM on grid environments was reported in [18]. Simulating the head-neck area of a patient using CT images with $10^{8}$ primary protons, the average turnaround time was approximately 20 minutes when the job was divided into 400 tasks. Therefore, the turnaround time for simulating $9 \times 10^{8}$ primary protons is approximately 180 minutes for 400 tasks.

\section{Dose verification}

Verification of the dose distributions in water has been performed using a water phantom from CT images. The voxel size was transformed to $2.0-\mathrm{mm}$ cubic voxels in the PTSIM from the original size. Each HU value was converted to the mass density of water by referring to a predefined conversion table. The converted density was rounded to a step resolution of $0.01 \mathrm{~g} / \mathrm{cm}^{3}$.

We verified the dose distributions in the treatment port of the proton gantry nozzle at the FPHPTC, which consists of a lateral spreading system, range compensators, monitors, and collimators. The lateral spreading system uses wobbler magnets and a lead scatter to expand a narrow beam to a broad beam. A ridge filter constructed from aluminum is used to provide energy modulation for forming the spread-out Bragg peak (SOBP).

We created a series of RT-IonPlan data to verify the effect of each beam device on dose distributions. The RT-IonPlan data were used for dose calculation in the PTSIM, K2Dose, and measurements, respectively. The preliminary depth-dose distributions are shown in Figure 3. The figure includes the SOBPs for $150 \mathrm{MeV}$, $190 \mathrm{MeV}$, and $235 \mathrm{MeV}$ primary proton beam energies, and ridge filters for SOBP ranging from $30 \mathrm{~mm}$ to 120 $\mathrm{mm}$. These results are preliminary and are shown only as an example. Therefore, a discussion of the dose distribution results is beyond the scope of the present paper.

Verification of dose-to-water in actual patient geometry has been performed at the HIBMC. The anatomical sites selected for the comparison were the head/neck, the liver, the lungs, and the pelvic region, all 
of which are typical anatomical sites for proton therapy. Discrepancies were observed around the distal fall-off regions. Detailed results and a discussion are provided in [19].

Dose-to-tissue distributions in patient geometry have been investigated in order to convert the divided HU spaces into material groups. Only a small difference was observed in the dose-to-water comparison [13]. The
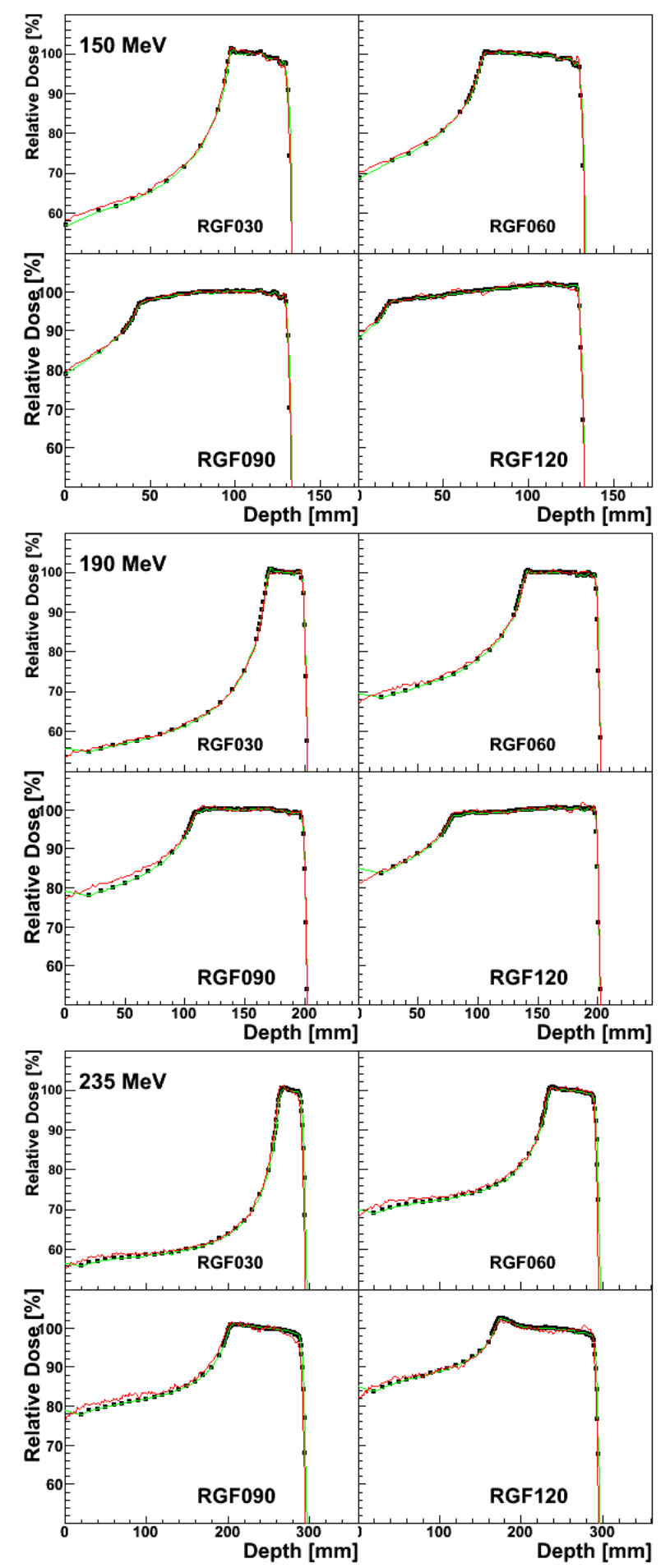

Figure 3. Preliminary results for depth-dose distributions in water for measurements (black point), K2Dose (green line), and PTSIM (red line), respectively. results agree with those reported [5] by $\mathrm{H}$. Paganetti et al. Simulations using segmented data of organs are considered.

\section{Conclusion}

The PTSIM is coordinated with the hospital information system and so is ready for clinical applications. Dose calculations using the PTSIM follow the procedure in the clinical treatment planning system. Therefore, medical staff can use the PTSIM without learning additional software in order to configure the treatment port or dose review.

The computational efficiency is still critical. However, UGI provides stable computing resources on multi-grid environments.

Since the most important role of Monte Carlo simulation in clinical applications is dose calculation based on particle interactions with human tissues, the exact material composition and weight of human tissues must be introduced in the simulation. The PTSIM provides two options for calculating dose-to-tissue in patient geometry. Studies involving systematic analysis are expected in order to reveal new aspects of dose distributions and improve treatment plans.

\section{Acknowledgements}

The authors would like to thank the members of the Geant4 collaboration, the National Institute of Informatics, and the Global Scientific Information and Computing Center of Tokyo Institute of Technology. The PTSIM was supported by JST CREST. The present study was supported in part by a grant from JSPS KAKENHI (No. 23540350 and No. 23390308).

\section{References}

[1] S. Agostinelli, J. Allison, K. Amako et al., Geant4 a simulation toolkit, Nucl. Instr. Meth. Phys. Res. A506 (2003), pp.250-303.

[2] J. Allison, K. Amako, J. Apostolakis et al., Geant4 developments and applications, IEEE Trans. Nucl. Sci. 53 No. 1 (2006), pp. 270-278.

[3] H. Paganetti, Monte Carlo method to study the proton fluence for treatment planning, Med. Phys. 25 (1998), pp.2370-2375.

[4] T. Aso, A. Kimura, S. Tanaka, H. Yoshida, N. Kanematsu. T. Sasaki and T. Akagi, Verification of the dose distributions with GEANT4 simulation for proton therapy, IEEE Trans. Nucl. Sci. 52 Issue 4 (2005), pp.896-902.

[5] H. Paganetti, H. Jiang, K. Parodi, R. Slopsema and M. Engelsman, Clinical implementation of full Monte Carlo dose calculation in proton beam therapy, Phys. Med. Biol. 53(17) (2008), pp.4825-4853.

[6] S. Kameoka. K. Amako, G. Iwai, K. Murakami, T. Sasaki, T. Toshito, T. Yamashita, T. Aso, A. 
Kimura, T. Kanai, N. Kanematsu, M. Komori, S. Takei, M. Tashiro, H. Koikegami, H. Tomita and T. Koi, Dosimetric evaluation of nuclear interaction models in the Geant4 Monte Carlo simulation toolkit for carbon-ion radiotherapy, Radiol. Phys. Technol. 1 (2008), pp.183-187.

[7] T. Aso, A. Kimura, S. Kameoka, K. Murakami, T. Sasaki and T. Yamashita, GEANT4 based simulation framework for particle therapy system, Conf. Rec. on IEEE NSS/MIC (2007), pp.2564-2567.

[8] T. Akagi, T. Aso, B. Faddegon, A. Kimura, N. Matsufuji, T. Nishio, C. Omachi, H. Paganetti, J. Perl, T. Sasaki, D. Sawkey, J. Schumann, J. Shin, T. Toshito, T. Yamashita and H. Yoshida, The PTSim and TOPAS projects bringing Geant4 to the particle therapy clinic, Prog. Nucl. Sci. Technol. 2 (2011), pp.912-917.

[9] L. Hong, M. Goietein, M. Bucciolini, R. Comiskey, B. Gottschalk, S. Rosenthal, C. Serago and M. Urie, A pencil beam algorithm for proton dose calculations, Phys. Med. Biol. 41 (1996), pp.1305-1330.

[10]DICOM, NEMA, Available: http://medical.nema.org/, [online].

[11]T. Aso, A. Kimura and T. Yamashita, Optimization of patient geometry based on CT data in GEANT4 for medical application, Nucl. Sci. Symp. Conf. Rec. IEEE, (2007), pp.2576-2580.

[12]W. Schneider, T. Bortfeld and W. Schlegel,
Correlation between CT numbers and tissue parameters needed for Monte Carlo simulations of clinical dose distributions, Phys. Med. Biol. 45 (2000), pp.459-478.

[13]T. Aso, A. Taniuchi, T. Yamashita, T. Akagi, C. Omachi and T. Sasaki, Estimation of influence of material assignment in CT data for hadron therapy using GEANT4, Nucl. Sci. Symp. Conf. Rec. IEEE NSS/MIC, (2010), pp.1835-1838.

[14]K. Kanematsu, T. Akagi, Y. Futami, A. Higashi, T. Kanai, N. Matsufuji, H. Tomura and H. Yamashita, A proton dose calculation code for treatment planning based on the pencil beam algorithm, Jap. J. Med. Phys. 18-1 (1998), pp.88-103.

[15]SAGA, OGF document GFD-R-P.90, Available: http://saga.cct.lsu.edu/, [online].

[16]RNS, OGF, Available: http://www.ggf.org/, [online].

[17]XML-RPC, Available: http://xmlrpc.scripting.com/, [online].

[18]T. Aso, R. Noto, G. Iwai, W. Takase and T. Sasaki, Particle therapy simulation framework on GRID environments, Proc. $12^{\text {th }}$ IEEE/ACM Int. Conf. on Grid Comp. (GRID2011) (2011), pp.222-223.

[19] T. Yamashita, T. Akagi, T. Aso, A. Kimura and T. Sasaki, Effect of inhomogeneity in patient's body on the accuracy of pencil beam algorithm in comparison to Monte Carlo, Phys. Med. Biol. 57 (2012), pp.7673-7689. 